\title{
ARTICLE Ketamine normalizes subgenual cingulate cortex hyper-activity in depression
}

\author{
Laurel S. Morris ${ }^{1}$, Sara Costi ${ }^{1}$, Aaron Tan ${ }^{1}$, Emily R. Stern ${ }^{2}$, Dennis S. Charney ${ }^{3}$ and James W. Murrough ${ }^{1,4}$
}

Mounting evidence supports the rapid antidepressant efficacy of the $N$-methyl-D-aspartate receptor antagonist, ketamine, for treating major depressive disorder (MDD); however, its neural mechanism of action remains poorly understood. Subgenual anterior cingulate cortex (sgACC) hyper-activity during rest has been consistently implicated in the pathophysiology of MDD, potentially driven in part by excessive hippocampal gluatmatergic efferents to sgACC. Reduction of sgACC activity has been associated with successful antidepressant treatment. This study aimed to examine whether task-based sgACC activity was higher in patients with MDD compared to controls and to determine whether this activity was altered by single-dose ketamine. In Study 1 , patients with MDD $(N=28)$ and healthy controls $(N=20)$ completed task-based functional magnetic resonance imaging using an established incentive-processing task. In Study 2, a second cohort of patients with MDD $(N=14)$ completed the same scanning protocol at baseline and following a $40 \mathrm{~min}$ infusion of ketamine $(0.5 \mathrm{mg} / \mathrm{kg})$. Task-based activation of sgACC was examined with a seed-driven analysis assessing group differences and changes from pre to post treatment. Patients with MDD showed higher sgACC activation to positive and negative monetary incentives compared to controls, associated with anhedonia and anxiety, respectively. In addition, patients with MDD had higher resting-state functional connectivity between hippocampus and sgACC, associated with sgACC hyper-activation to positive incentives, but not negative incentives. Finally, ketamine reduced sgACC hyper-activation to positive incentives, but not negative incentives. These findings suggest a neural mechanism by which ketamine exerts its antidepressant efficacy, via rapid blunting of aberrant sgACC hyper-reactivity to positive incentives.

Neuropsychopharmacology (2020) 45:975-981; https://doi.org/10.1038/s41386-019-0591-5

\section{INTRODUCTION}

Major depressive disorder (MDD) is one of the world's largest public health issues to date, representing the leading cause of disability worldwide [1]. Despite its widespread prevalence, widely prescribed pharmacological treatments such as selective serotonin re-uptake inhibitors (SSRI) are slow to act and are often only partially effective, rendering a significant proportion of patients unresponsive $[2,3]$. The US Food and Drug Administration has recently approved the s-enantiomer of ketamine, 'esketamine' for the treatment of depression in patients with MDD who have failed to respond to prior antidepressants, following growing clinical evidence of the efficacy of ketamine over the past two decades [4-6]. However, while the first report of the antidepressant effect of ketamine was published in 2000, there remains a lack of clarity regarding its effect on neural functioning in patients with MDD.

MDD is associated with widespread neural, cognitive, and behavioral dysfunction. One of the most consistently implicated regions in MDD is the subgenual anterior cingulate cortex (sgACC), putatively corresponding to the infralimbic cortex in rodent models [7], although functional homology has not been widely demonstrated between human and rodent regions. The human sgACC plays an important role in emotion regulation [8], reward anticipation [9], and anhedonia (inability to feel pleasure) [10, 11], core features of MDD. The sgACC is hyper-active in MDD [12] and reducing sgACC hyper-activity has been associated with reduction in depressive symptoms [13]. Evidence from rodent models suggests that aberrant infralimbic cortex hyper-activity might be driven by excessive hippocampal output, leading to depressive symptoms or behaviors [14]. Indeed, chronic mild stress in rats leads to increased hippocampal extracellular glutamate [15] and output particularly to the infralimbic cortex $[14,16]$. The hippocampus and sgACC are strongly anatomically connected in humans [17] and several studies in MDD demonstrate hippocampus and sgACC hyper-connectivity [18-20], associated with illness duration and severity $[18,20]$.

A recent study in non-human primates has demonstrated a critical link between sgACC hyper-activation, core depressive symptoms, and the antidepressant effects of ketamine [11]. Overactivation of the sgACC (Brodmann area 25 ) via glutamate re-uptake inhibition led to deficits in anticipatory arousal to receipt of a primary reinforcer (food reward) in marmosets-a model analog of anhedonia in MDD [11]. In this model, single-dose ketamine led to an improvement in anticipatory arousal, as well as a normalization of sgACC hyper-activity, whereas SSRI treatment had no effect [11]. In this study, we aimed to determine the responsivity of sgACC to receipt of a secondary reinforcer (money) in humans with MDD and to determine whether the rapid antidepressant effect of ketamine

\footnotetext{
${ }^{1}$ Depression and Anxiety Center for Discovery and Treatment, Department of Psychiatry, Icahn School of Medicine of Mount Sinai, New York, NY, USA; ${ }^{2}$ New York University School of Medicine, New York, NY and Nathan S. Kline Institute, Orangeburg, NY, USA; ${ }^{3}$ Office of the Dean, Icahn School of Medicine of Mount Sinai, New York, NY, USA and ${ }^{4}$ Department of Neuroscience, Icahn School of Medicine at Mount Sinai, New York, NY, USA Correspondence: James W. Murrough (james.murrough@mssm.edu)
}

Received: 27 September 2019 Revised: 15 November 2019 Accepted: 10 December 2019 Published online: 2 January 2020 
may act by altering sgACC activation. We also aimed to replicate previous reports of hippocampal-sgACC hyper-connectivity in patients with MDD, and to determine the relationship between resting connectivity and task-based activation.

\section{METHODS AND MATERIALS}

Participants and procedures

Study 1 and 2. Subjects in Study 1 and Study 2 were recruited at the Icahn School of Medicine at Mount Sinai and underwent a complete medical and psychiatric assessment. For Study 1, subjects were recruited between September 2016 and August 2018; for Study 2, subjects were recruited between June 2013 and September 2016. Depressed patients meeting Diagnostic and Statistical Manual of Mental Disorders (DSM) criteria for a depressive disorder (MDD, persistent depressive disorder, or other specified depressive disorder) according to the Structured Clinical Interview for DSM Axis I Disorders-Patient Edition (SCID-I/P) were deemed eligible to participate in the study. Subjects were excluded if they had substance use disorder in the past 2 years, or a lifetime history of a psychotic disorder, bipolar disorder, a developmental disorder, obsessive-compulsive disorder, an eating disorder, or a personality disorder as their primary presenting problem. Subjects were free of antidepressant or other psychotropic medication at the time of the scans. Healthy control subjects were free of any current or past psychiatric disorder, as determined by the SCID-I/P.

Study 2. Subjects in Study 2 were enrolled in a clinical trial (clinicaltrials.gov ID: NCT01880593) between June 2013 and September 2016, in which they received intravenous ketamine in a prospective, open-label design (for detailed methods, see [21]). Subjects were eligible if they had previously failed to respond to at least two previous antidepressant medications, as determined by the antidepressant treatment history form (ATHF). Subjects were excluded if they had any history of recreational use of ketamine or phencyclidine. Subjects received one intravenous ketamine infusion $(0.5 \mathrm{mg} / \mathrm{kg}$ over $40 \mathrm{~min})$. Pre- and post-ketamine magnetic resonance imaging (MRI) scans were collected within 14 and 5 days of this single infusion, respectively.

Depression severity was assessed using the MontgomeryAsberg Depression Rating Scale (MADRS) [22]. All subjects also completed the self-reported temporal experience of pleasure scale (TEPS) [23] to assess anticipatory anhedonia and the state-trait inventory of cognitive and somatic anxiety (STICSA) [24] to assess trait anxiety. The TEPS yields a total score for anhedonia, as well as sub-scores for anticipatory and consummatory anhedonia, respectively. All subjects provided written informed consent prior to any study procedures using consent forms approved by the Program for the Protection of Human Subjects at Mount Sinai for Study 1 and Study 2.

\section{MRI acquisition and processing}

All MRI data was acquired with a Siemens 3T MAGNETOM Skyra scanner and a 32-channel head coil at Mount Sinai's Translational and Molecular Imaging Institute. Scans included an anatomical T1weighted scan, a functional scan during resting state (10 min, eyes open), and a task-based functional scan with the incentive flanker task (IFT, detailed below).

Study 1. The anatomical T1-weighted images were acquired with a magnetization-prepared 2 rapid gradient-echo sequence, which collects two volumes after each inversion for improved image quality (repetition time $(T R)=4000 \mathrm{~ms}$, echo time $(T E)=1.9$, inversion $1 / 2$ time $=633 / 1860$, field of view $(F O V)=186 \times 162$, voxel resolution $=1 \times 1 \times 1 \mathrm{~mm}^{3}$ ), and the functional scans for both resting state and task performance were collected with a multi-echo (ME) multi-band accelerated echo planar imaging sequence $(\mathrm{TR}=882 \mathrm{~ms}$, $\mathrm{TE}=11.0,29.7,48.4,67.1$, multi-band factor $=5, \quad F O V=560 \times 560$, voxel resolution $=3 \times 3 \times 3 \mathrm{~mm}^{3}$, flip angle $=45$ ). Both functional scans were preprocessed and denoized for motion and physiological noise using MEindependent component analysis (ME-ICA) [25, 26]. ME functional MRI data was decomposed into independent components, and scaled against TE $[25,26]$. Components with high TE dependence are considered BOLD like, whereas components with low TE dependence are considered noise like $[25,26]$. Removal of nonBOLD components allows robust data denoizing for motion, physiological, and scanner artifacts $[25,26]$.

Study 2. The anatomical T1-weighted MPRAGE images were acquired with the following acquisition parameters: TR $=2400 \mathrm{~ms}$, $\mathrm{TE}=2.07 \mathrm{~ms}$, flip angle $=8^{\circ}$, and bandwidth $=240$. Functional scans for both resting state and task performance were collected with a multi-band gradient-echo echo planar sequence with 70 axial slices $(T R=1000 \mathrm{~ms}, T E=35 \mathrm{~ms}$, multi-band factor $=7$, bandwidth $=1860, \quad \mathrm{FOV}=756 \times 864$, voxel resolution $=2.1 \times$ $2.1 \times 2.1$, flip angle $=60$ ). Functional data was despiked (3dDespike), outliers removed (3dToutcount), and subject to rigid-body registration (3dvolreg) using a combination of AFNI [27] and FSL [28]. Motion derivatives were used as regressor of no interest in subsequent first-level analyses. Resting-state data was additionally denoized using ICA-based correction, bandpass filtered (0.01-0.1 $\mathrm{Hz}$ ), and detrended. Functional data was smoothed with a $5 \mathrm{~mm}$ kernel. All denoized functional data was coregistered with their respective $\mathrm{T} 1$ and normalized to a standard Montreal Neurological Institute template using Advanced Normalization Tools (http://www.picsl.upenn.edu/ANTs) with diffeomorphic symmetric normalization transformation registration.

\section{Incentive flanker task}

All subjects completed the IFT (see Fig. 1), a modified version of the monetary incentive delay task, described in detail elsewhere [29,30], which allows participant engagement due to a cognitive task, as well as measurement of neural activity related to both cue presentation and performance-based feedback. There were three trial types (reward/loss/neutral). Each trial contained an initial monetary cue signaling the value of the current trial (reward/loss/ neutral) (2-6 s). This was followed by a flanker task, which consisted of a display of five letters in row and subjects were instructed to respond to the middle letter only by pressing a left button if the middle letter was $\mathrm{S}$ or $\mathrm{K}$, and the right button if the middle letter was $\mathrm{H}$ or C. Flanker letters could be congruent or incongruent $(50 / 50 \%)$ with the middle letter. The response period was titrated based on baseline performance (the mean baseline performance was multiplied by 1.5 , with $1700 \mathrm{~ms}$ maximum). A correct response was the correct button press within the response period. After a button response, feedback was displayed for $2 \mathrm{~s}$. Across all trial types, feedback following a correct response was a smiling cartoon face with the word: "Correct!," whereas feedback following an incorrect response was a frowning cartoon face with: "Incorrect!." These were considered positive and negative feedback, respectively, collapsed across trial types. In addition, feedback included monetary value (correct response on reward/loss/neutral $=\$ 0.50 / \$ 0.00 / \$ 0.00$; incorrect response on reward/loss/neutral $=\$ 0.00 /-\$ 0.50 / \$ 0.00)$, and at the end of the session, participants were rewarded with real money based on their performance. One-third of cues were followed by a blank screen for $2 \mathrm{~s}$ (instead of the flanker stimuli) to break collinearity between the cue and feedback. Outcome feedback was followed by a blank inter-trial interval for 2-6s before the next trial. There were 40 of each trial type (reward/loss/neutral), producing a total of 120 trials. Trials were presented in pseudorandom order and equally divided across four runs of $\sim 6$ min each.

Data analysis and statistics

Task-based fMRI. Task-based fMRI data was processed in the same way for both cohorts of subjects. First-level general linear 

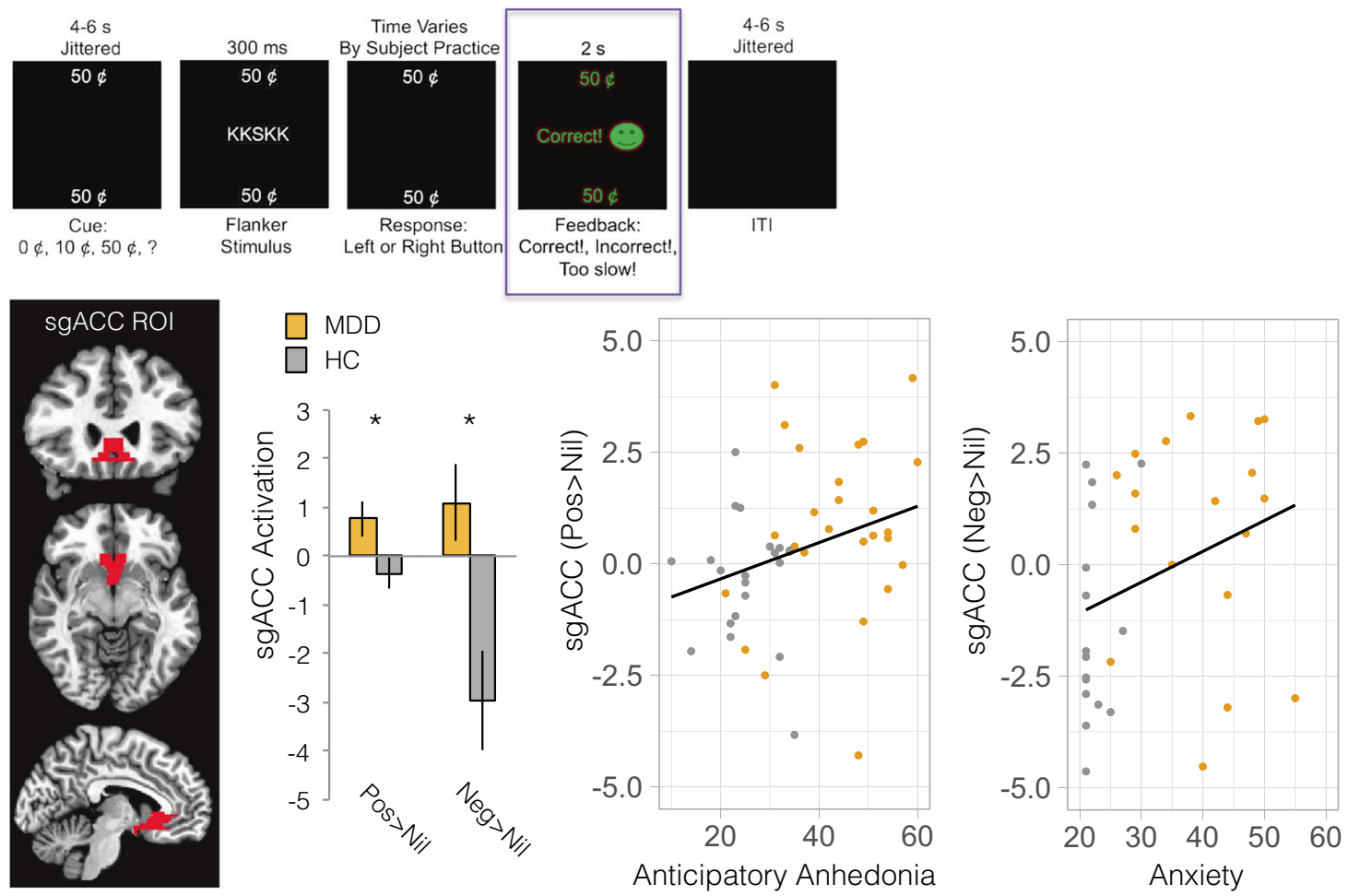

Fig. 1 Linking subgenual anterior cingulate cortex (sgACC) activation with distinct symptoms of depression. Top: Subjects completed the incentive flanker task (IFT) during functional MRI. The task consisted of reward, loss, and neutral trials, which included a cue, followed by a flanker task (congruent/incongruent), followed by feedback (positive/negative). Positive and negative feedback was contrasted against no feedback (Nil). Bottom: Patients with major depressive disorder (MDD) showed higher activity within the sgACC region of interest (ROI) compared to healthy controls (HCs) to both positive $\left(T_{(45)}=2.208, p=0.032\right)$ and negative $\left(T_{(42)}=3.036, p=0.004\right)$ feedback. Higher sgACC activation to positive was associated with anhedonia $(R=-0.34, p=0.028)$, whereas higher activation to negative was associated with higher anxiety $(R=0.386, p=0.012)$ across the full cohort.

models included regressors for cue onset (reward/loss/neutral), flanker onset, and feedback onset (positive/negative), each including duration modulation using AFNI's dmBLOCK function and convolved with the hemodynamic response function. Contrasts of interest included gain and loss cues, contrasted against the neutral cue, and positive and negative feedback, contrasted against a blank screen that followed the cue, which represented "no outcome." Activation for each contrast was extracted within the sgACC ROI (based on Brodmann area 25) for each subject and entered into independent-samples $t$ test for group difference comparison, controlling for age and sex, and paired-samples $t$ test for computing pre/post-ketamine differences. Pearson's correlation was used for examination of relationships between sgACC activation and clinical symptoms.

Resting-state fMRI. Functional connectivity was computed between the sgACC and hippocampus ROl's (automated anatomical labeling atlas) for all subjects using Pearson's correlation and using Z-score normalization. Normalized functional connectivity $Z$-values were entered into independent-samples $t$ test for group difference comparison, controlling for age and sex, and Pearson's correlation was used for examination of relationships with sgACC task-based activation.

\section{RESULTS}

Participants

Participant characteristics are summarized in Table 1. For Study 1, 28 MDD subjects and $20 \mathrm{HC}$ subjects completed the scan.

\begin{tabular}{|llll}
\hline Table 1. Subject characteristics for Study 1 and Study 2. & \\
\hline & MDD & HC & $p$ Value \\
\hline Study 1 & & & \\
$\quad$ Female/male & $14 / 14$ & $7 / 13$ & \\
Age (SD) & $36.5(11)$ & $37.8(9.4)$ & 0.38 \\
MADRS & $27.96(5.5)$ & $1(1.6)$ & $<0.001$ \\
Anhedonia (anticipatory) & $26.46(10.8)$ & $45.0(6.7)$ & $<0.001$ \\
Anhedonia (consummatory) & $25.61(10.3)$ & $39.2(6.2)$ & $<0.001$ \\
Anxiety & $23.79(7.5)$ & $11.15(2.2)$ & $<0.001$ \\
Study 2 & & & \\
Female/male & $9 / 7$ & & \\
Age (SD) & $44.69(11.4)$ & & \\
MADRS (pre-ketamine) & $32.13(5.1)$ & & \\
MADRS (post-ketamine) & $15.29(7.5)$ & & \\
Anhedonia (pre-ketamine) & $35.69(6.0)$ & & \\
Anhedonia (post-ketamine) & $28.94(5.6)$ & & \\
\hline
\end{tabular}

Subject characteristics are reported for patients with MDD and HCs. Depression severity was measured using the MADRS. Anhedonia was measured using the TEPS and anxiety with the STICSA. For Study 1, scores for the anticipatory and consummatory sub-scales of the TEPS are displayed; For Study 2, the TEPS total score is displayed

$M D D$ major depressive disorder, $H C$ healthy controls, MADRS MontgomeryAsberg Depression Rating Scale, TEPS temporal experience of pleasure scale, STICSA state-trait inventory of cognitive and somatic anxiety 
For Study 2, MDD subjects received one 40-min ketamine infusion $(0.5 \mathrm{mg} / \mathrm{kg})$, with both pre- and post-ketamine MRI data available for 14 MDD subjects.

Incentive flanker task

Functional MRI data during the IFT (see Fig. 1) were processed and analyzed for $28 \mathrm{MDD}$ subjects (age $=36.5 \pm 11,14$ female) and 20 $\mathrm{HC}$ subjects (age $=37.8 \pm 9.4,7$ female). One MDD subject was excluded due to excessive motion during MRI. Groups did not differ in their performance accuracy (MDD $=85.9 \pm 10.4 \%$, $\mathrm{HC}=87.7 \pm 10.1 \%, p=0.76)$, or their baseline reaction times $(\mathrm{MDD}=927.6 \pm 172.5 \mathrm{~ms}, \mathrm{HC}=859.3 \pm 145.7 \mathrm{~ms}, p=0.16)$. Two MDD subjects and one HC subject performed $100 \%$ accuracy and therefore received no negative feedback, and thus were not available for analyses including negative feedback.

MDD subjects showed sgACC hyper-activity to receipt of positive and negative feedback, compared to $\mathrm{HC}$ (positive, $T_{(45)}=2.208, p=0.032$; negative, $T_{(42)}=3.036, p=0.004$; Fig. 1). sgACC hyper-activity to receipt of positive feedback was associated with worse anticipatory anhedonia $(R=-0.34, p=0.028$, Fig. 1), and not anxiety $(R=0.129, p=0.416$; difference in correlations: $Z=-1.9, p=0.05$ ), across the full sample (controlling for age, sex). This correlation remained significant when also controlling for anxiety $(R=-0.323, p=0.020)$. In contrast, sgACC hyper-activity to receipt of negative feedback was associated with worse anxiety $(R=0.386, p=0.012$, Fig. 1$)$, and not with anticipatory anhedonia $(R=-0.282, p=0.07$; difference in correlations: $Z=2.8, p=$ 0.004 ), across the full sample (controlling for age, sex). This remained significant when also controlling for anticipatory anhedonia $(R=-0.290, p=0.033)$. sgACC hyper-activity to positive did not correlate with sgACC hyper-activity to negative $(R=0.167, p=0.29)$, suggesting that they may be dissociable. There were no group differences in sgACC activation to gain or loss vs. neutral cue contrasts ( $\left.p^{\prime} s>0.2\right)$.

Resting-state functional MRI

Resting-state functional MRI data were processed and analyzed for the same $28 \mathrm{MDD}$ subjects (age $=36.5 \pm 11,14$ female) and $20 \mathrm{HC}$ subjects (age $=37.8 \pm 9.4,7$ female). Patients with MDD had increased resting-state functional connectivity between the hippocampus and sgACC, compared to controls $\left(t_{(44)}=-2.2\right.$, $p=0.031$, Fig. 2). Hippocampus and sgACC connectivity was positively correlated with sgACC hyper-activation to positive feedback ( $R=0.33, p=0.028$, Fig. 2$)$, but not negative feedback
$(R=-0.15, p=0.924 ;$ difference in correlations: $Z=2.6, p=0.007)$ across the full sample.

Effects of ketamine

There was a significant improvement in MDD symptoms following a single-dose intravenous ketamine (MADRS, $T_{(16)}=10.52, p<$ $0.0001)$, as well as improvements in anhedonia $\left(T_{(16)}=5.52, p<\right.$ 0.0001) (see Table 1).

Single-dose ketamine significantly reduced sgACC activation to positive feedback $\left(t_{(13)}=3.17, p=0.007\right.$, Fig. 3$)$, but not negative feedback $\left(t_{(13)}=1.42, p=0.179\right.$, repeated-measures ANOVA: time $\times$ feedback interaction $\left.F_{(1,13)}=6.09, p=0.028\right)$. Interestingly, higher pre-ketamine sgACC activation to positive feedback was associated with a better improvement in anhedonia after ketamine $(R=-0.437, p=0.045$, Fig. 3). There was no significant change in hippocampus and sgACC connectivity between pre and post ketamine $(p>0.05)$.

Subdivisions within sgACC

Given that the sgACC ROI encompassed a broad region, we performed an exploratory analysis dividing the sgACC into subdivisions corresponding to the architectonic boundaries of (1) area 25 (posterior medial wall) and (2) prelimbic area 32 (PL32) according to Ongur et al. [31] (see Fig. 4). We found that area PL32 was significantly hyper-active to negative feedback, but not positive feedback $(p=0.048, p=0.272)$, whereas area 25 was trending towards significant hyper-activation to positive feedback, but not negative feedback ( $p=0.054, p=0.478$, Fig. 4). Furthermore, area PL32 hyper-activity to negative feedback (but not positive feedback) was correlated with anxiety (cognitive, $R=0.359, p=0.017$, Fig. 4) and not anticipatory anhedonia $(R=$ $-0.207, p=0.178$; difference in correlations: $Z=2.3, p=0.019$ ), whereas area 25 hyper-activity to positive feedback (but not negative feedback) was correlated with anticipatory anhedonia $(R=-0.346, p=0.017$, Fig. 4$)$ and not anxiety $(R=0.15, p=0.922$; difference in correlations: $Z=-2.1, p=0.035$ ). This suggests an anatomical-functional dissociation whereby area PL32 (anterior sgACC) processing of negative feedback is linked with anxiety, while area 25 (posterior sgACC) processing of positive feedback is linked with anticipatory anhedonia.

Hippocampus and sgACC connectivity was correlated with area 25 hyper-activity to positive feedback only $(R=0.260, p=$ 0.045 ) and not with negative or PL32 with either ( $p$ 's >0.05). An exploratory analysis of hippocampal connectivity with area 25
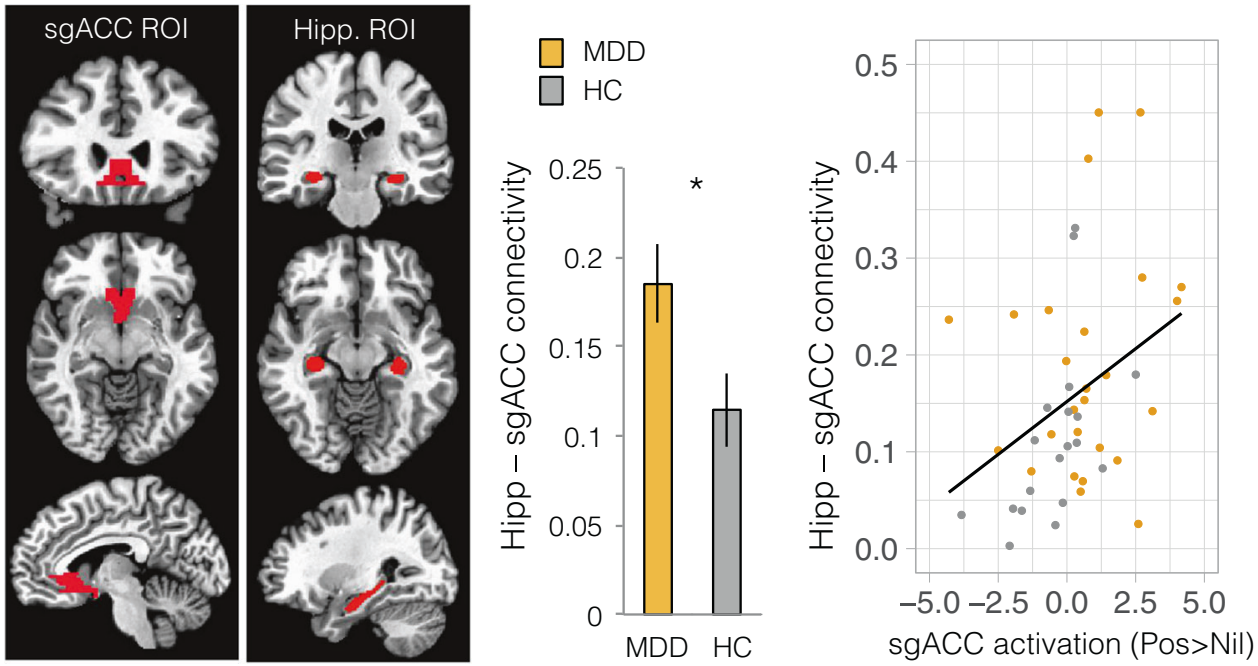

Fig. 2 Hippocampus and subgenual anterior cingulate cortex (sgACC) connectivity in depression. Patients with major depressive disorder (MDD) had increased resting-state functional connectivity between the hippocampus and sgACC, compared to healthy controls $\left(\mathrm{HCs}, t_{(44)}=-2.2, p=0.031\right)$. This connectivity was associated with higher sgACC activation to positive feedback $(R=0.33, p=0.028)$. 
and PL32 specifically revealed that connectivity between hippocampus and both area 25 and PL32 were correlated with area 25 hyper-activity to positive feedback ( $p=0.046, p=0.035)$, and not negative feedback ( $p=0.105, p=0.308)$, and only hippocampal connectivity with PL32 correlated with anxiety $(p=0.042)$. Finally, there was a trend towards ketamine specifically reducing area 25 hyper-activity to positive feedback ( $t=2.045, p=0.062)$, but not area PL32 hyper-activity to negative feedback $(p=0.74)$.

Pre-Ketamine

Post-Ketamine
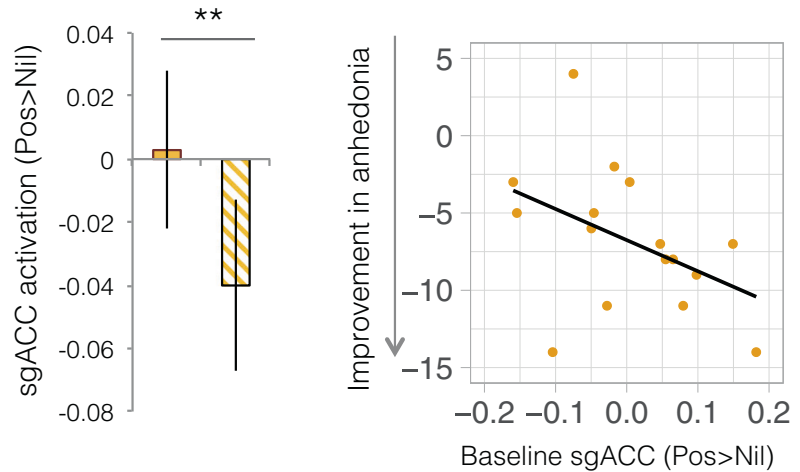

Fig. 3 The influence of ketamine on subgenual anterior cingulate cortex (sgACC) activation. Ketamine reduced sgACC activation to positive feedback in patients MDD $\left(t_{(13)}=3.17, p=0.007\right)$. Greater pre-ketamine sgACC activation was associated with greater improvement in anhedonia (TEPS total score) after single-dose ketamine $(R=-0.437, p=0.045)$.

\section{DISCUSSION}

These data are consistent with previous studies in animal models demonstrating excessive stress-related glutamatergic signalling in projections from hippocampus to sgACC, sgACC hyper-activity, and links with anhedonic behaviors [12-14]. We demonstrate a double dissociation whereby sgACC hyper-activation to positive feedback is associated with anhedonia, whereas hyper-activation to negative feedback is associated with anxiety. In addition, we show that ketamine blunts sgACC hyper-activation to positive feedback, but not negative feedback in patients with MDD. Finally, we highlight a potential anatomical distinction within sgACC, whereby posterior area 25 is more related to positive processing and anhedonia, whereas anterior PL32 is more associated with negative processing and anxiety.

This translational finding mirrors a recent study in marmosets also demonstrating that single-dose ketamine led to normalization of sgACC hyper-activity and an improvement in an autonomic measure of anhedonia (anticipatory arousal) [11]. The current findings coincide with previous reports of increased resting sgACC activity in MDD $[12,13]$ and extends this work by implicating hyper-activity during valence processing in a task-based setting additionally. We further highlight the potential functional heterogeneity within the sgACC region, suggesting that anhedonia and anxiety may be separately related to area 25 and PL32 as defined by Ongur et al. [31], respectively. This is in line with evidence of phasic reward value encoding in more posterior, ventral subgenual regions in non-human primates [32]. It is important to note that while this work utilizes definition of PL32 as defined by Ongur et al. [31], other work provides alternative subdivisions of sgACC, including, for example, a larger region of area 25 in macaques, which is largely separated from area 32 by area 24 [33]. Therefore, refining and comprehensively characterizing the 
980

specific valence-processing functions of subdivisions within sgACC will be critical for tackling the heterogeneity of MDD.

This work additionally underscores the functional role of hippocampal projections to area 25 , which have been previously shown to be hyper-active in rodents following chronic mild stress [14-16], and related to MDD illness duration and severity in humans $[18,20]$. A recent study has also demonstrated that interactions between anterior hippocampus to area 25 are critical for normal harm avoidance behaviors [34], a common indication of anxiety-like behaviors in humans with depression, as well as often co-morbid anxiety disorders [35, 36].

Previous findings of effects of ketamine on neural activity have been mixed. One study of acute ketamine administration in a small sample of healthy male subjects resulted in reduced sgACC resting functional connectivity with hippocampus, thalamus, and brainstem [37]. Another study of single-dose ketamine showed no change in whole brain metabolism as measured by positron-emission tomography, but showed both increases and decreases in regional resting metabolism throughout the cortex, with clinical improvement associated with reduced parahipp/temporoparietal metabolism [38]. Similar to the current findings, another previous study demonstrated that ketamine decreased ventromedial prefrontal/sgACC activity in healthy male subjects [39]. However, the same group also showed the opposite effect in patients with MDD [40]. In the latter study, patients with MDD were given slow, single-dose ketamine (non-bolus), which acutely increased sgACC resting activity as measured using pharmacological MRI, although it showed no antidepressant effect [40]. This suggests that the effect of ketamine on neural activity may depend on the antidepressant efficacy, in which a clinical effect is associated with reduced sgACC activation. Since the methodology between these two studies were quite different (pharmacological MRI versus task-based functional MRI), further studies are required to explain these inconsistencies.

Interestingly, the current findings revealed altered sgACC activation to valenced feedback rather than cue. Outcome anticipation (cue) rather than outcome receipt (feedback) is typically related to sgACC hyper-activation in primate work [11]. However, this inconsistency may be due to differences in rewards used in humans and primate studies: in humans, money is used as a secondary reinforcer rather than the primary food reinforcers typically used in primate studies [11]. Since money is a secondary reinforcer [41, 42], receipt could be considered a cue-like event rather than a consummatory event.

There were several limitations of this study. First, the two studies were performed on two cohorts of patients, with differing gender make-up, so follow-up replication studies in the same subjects will be required. Second, the MRI scan pre and post ketamine did not happen on the same day as the infusion. This was to mitigate against any psychotomimetic effects of ketamine and to better characterize longer-term changes in mood. While there was a relatively long lag between the ketamine infusion and the post-ketamine scan (up to 14 days), previous studies have suggested that even single-dose ketamine can have longer-term effects on mood symptoms [43]. Further studies should explore the effects of multiple doses of ketamine on neural activity as well as examine short and longer-term effects of the drug.

The current work demonstrates that sgACC hyper-activity in depression is not just confined to resting state, but is observed during task too. We further dissect putative subregions within sgACC and indicate a more posterior area 25 -like region associated with positive incentive processing and anhedonia, versus a more anterior PL32-like region associated more with negative processing and anxiety. Furthermore, this work suggests that ketamine acts rapidly to normalize the area 25 hyper-activity to positive incentives, potentially indicating an antidepressant mechanism.

\section{FUNDING AND DISCLOSURE}

Funding was provided by the National Institute of Mental Health (Grants R21MH109771 and K23MH094707 to J.W.M.) and the Doris Duke Charitable Foundation (Grant 2013098 to J.W.M.). Additional funding for this study was provided by the Ehrenkranz Laboratory for Human Resilience, a component of the Depression and Anxiety Center for Discovery and Treatment at the Icahn School of Medicine at Mount Sinai. The content is solely the responsibility of the authors and does not necessarily represent the official views of the National Institutes of Health or other funding bodies. In the past 5 years, J.W.M. has provided consultation services to Otsuka, Clexio Biosciences, FSV7, Boehringer Ingelheim, Sage Therapeutics, Novartis, Allergan, Fortress Biotech, Janssen Research and Development, Genentech, Medavante-Prophase, and Global Medical Education (GME) and has received research support from Avanir Pharmaceuticals, Inc. J.W.M. is named on a patent pending for neuropeptide $Y$ as a treatment for mood and anxiety disorders. D.C. is named as co-inventor on patents filed by the Icahn School of Medicine at Mount Sinai (ISMMS) relating to ketamine for the treatment for treatment-resistant depression, suicidal ideation, and other disorders. ISMMS has entered into a licensing agreement with Janssen Pharmaceuticals, Inc. and it has and will receive payments from Janssen under the license agreement related to these patents for the treatment of treatment-resistant depression and suicidal ideation. Consistent with the ISMMS Faculty Handbook (the medical school policy), D.C. is entitled to a portion of the payments received by the ISMMS. Since SPRAVATO has received regulatory approval for treatment-resistant depression, ISMMS and thus, through the ISMMS, D.C. will be entitled to additional payments, beyond those already received, under the license agreement. D.C. is a named co-inventor on several patents filed by ISMMS for a cognitive training intervention to treat depression and related psychiatric disorders. The ISMMS has entered into a licensing agreement with Click Therapeutics Inc. and has and will receive payments related to the use of this cognitive training intervention for the treatment of psychiatric disorders. In accordance with the ISMMS Faculty Handbook, D.C. has received a portion of these payments and is entitled to a portion of any additional payments that the medical school might receive from this license with Click Therapeutics. D.C. is a named co-inventor on a patent application filed by the ISMMS for the use of intranasally administered neuropeptide $Y$ (NPY) for the treatment of mood and anxiety disorders. This intellectual property has not been licensed. D.C. is a named co-inventor on a patent application in the United States, and several issued patents outside the United States filed by the ISMMS related to the use of ketamine for the treatment of post-traumatic stress disorder (PTSD). This intellectual property has not been licensed The authors declare no competing interests.

\section{ADDITIONAL INFORMATION}

Publisher's note Springer Nature remains neutral with regard to jurisdictional claims in published maps and institutional affiliations.

\section{REFERENCES}

1. Collins PY, Patel V, Joestl SS, March D, Insel TR, Daar AS, et al. Grand challenges in global mental health. Nature. 2011:475:27-30.

2. Rush AJ, Trivedi MH, Wisniewski SR, Nierenberg AA, Stewart JW, Warden D, et al. Acute and longer-term outcomes in depressed outpatients requiring one or several treatment steps: a STAR*D report. Am J Psychiatry. 2006;163:1905-17.

3. Tollefson GD, Holman SL. How long to onset of antidepressant action: a metaanalysis of patients treated with fluoxetine or placebo. Int Clin Psychopharmacol. 1994;9:245-50.

4. Berman RM, Cappiello A, Anand A, Oren DA, Heninger GR, Charney DS, et al. Antidepressant effects of ketamine in depressed patients. Biol Psychiatry. 2000;47:351-4. 
5. Zarate CA Jr, Singh JB, Carlson PJ, Brutsche NE, Ameli R, Luckenbaugh DA, et al. A randomized trial of an $\mathrm{N}$-methyl-D-aspartate antagonist in treatment-resistant major depression. Arch Gen Psychiatry. 2006;63:856-64.

6. Murrough JW, losifescu DV, Chang LC, Al Jurdi RK, Green CE, Perez AM, et al. Antidepressant efficacy of ketamine in treatment-resistant major depression: a two-site randomized controlled trial. Am J Psychiatry. 2013;170:1134-42.

7. Sousa N. The dynamics of the stress neuromatrix. Mol Psychiatry. 2016;21:302-12.

8. Stevens FL, Hurley RA, Taber KH. Anterior cingulate cortex: unique role in cognition and emotion. J Neuropsychiatry Clin Neurosci. 2011;23:121-5.

9. Rudebeck PH, Putnam PT, Daniels TE, Yang T, Mitz AR, Rhodes SE, et al. A role for primate subgenual cingulate cortex in sustaining autonomic arousal. Proc Natl Acad Sci U S A. 2014;111:5391-6.

10. Keedwell PA, Andrew C, Williams SC, Brammer MJ, Phillips ML. The neural correlates of anhedonia in major depressive disorder. Biol Psychiatry. 2005;58:843-53.

11. Alexander L, Gaskin PLR, Sawiak SJ, Fryer TD, Hong YT, Cockcroft GJ, et al. Fractionating blunted reward processing characteristic of anhedonia by over-activating primate subgenual anterior cingulate cortex. Neuron. 2019;101:307-20 e6.

12. Drevets WC, Savitz J, Trimble M. The subgenual anterior cingulate cortex in mood disorders. CNS Spectr. 2008;13:663-81.

13. Mayberg HS, Lozano AM, Voon V, McNeely HE, Seminowicz D, Hamani C, et al. Deep brain stimulation for treatment-resistant depression. Neuron. 2005;45:651-60.

14. Hamilton JP, Chen G, Thomason ME, Schwartz ME, Gotlib IH. Investigating neural primacy in major depressive disorder: multivariate Granger causality analysis of resting-state fMRI time-series data. Mol Psychiatry. 2011;16:763-72.

15. MacQueen G, Frodl T. The hippocampus in major depression: evidence for the convergence of the bench and bedside in psychiatric research? Mol Psychiatry. 2011;16:252-64

16. Airan RD, Meltzer LA, Roy M, Gong Y, Chen H, Deisseroth K. High-speed imaging reveals neurophysiological links to behavior in an animal model of depression. Science. 2007;317:819-23.

17. Johansen-Berg H, Gutman DA, Behrens TE, Matthews PM, Rushworth MF, Katz E, et al. Anatomical connectivity of the subgenual cingulate region targeted with deep brain stimulation for treatment-resistant depression. Cereb Cortex. 2008;18:1374-83.

18. Zhang J, Wang J, Wu Q, Kuang W, Huang X, He Y, et al. Disrupted brain connectivity networks in drug-naive, first-episode major depressive disorder. Biol Psychiatry. 2011;70:334-42.

19. Sambataro F, Wolf ND, Pennuto M, Vasic N, Wolf RC. Revisiting default mode network function in major depression: evidence for disrupted subsystem connectivity. Psychol Med. 2014;44:2041-51.

20. de Kwaasteniet B, Ruhe E, Caan M, Rive M, Olabarriaga S, Groefsema M, et al. Relation between structural and functional connectivity in major depressive disorder. Biol Psychiatry. 2013;74:40-7.

21. Costi S, Soleimani L, Glasgow A, Brallier J, Spivack J, Schwartz J, et al. Lithium continuation therapy following ketamine in patients with treatment resistant unipolar depression: a randomized controlled trial. Neuropsychopharmacology. 2019.

22. Montgomery SA, Asberg M. A new depression scale designed to be sensitive to change. Br J Psychiatry. 1979;134:382-89.

23. Chan RC, Shi YF, Lai MK, Wang YN, Wang Y, Kring AM. The temporal experience of pleasure scale (TEPS): exploration and confirmation of factor structure in a healthy Chinese sample. Plos One. 2012;7:e35352.

24. Ree M, French D, MacLeod C, Locke V. Distinguishing cognitive and somatic dimensions of state and trait anxiety: Development and validation of the StateTrait Inventory for Cognitive and Somatic. Anxiety (STICSA). 2008;36:313-32.
25. Kundu P, Brenowitz ND, Voon V, Worbe $Y$, Vertes PE, Inati SJ, et al. Integrated strategy for improving functional connectivity mapping using multiecho fMRI. $P$ Natl Acad Sci USA. 2013;110:16187-92.

26. Kundu P, Inati SJ, Evans JW, Luh WM, Bandettini PA. Differentiating BOLD and non-BOLD signals in fMRI time series using multi-echo EPI. Neuroimage. 2012;60:1759-70

27. Cox RW. AFNI: software for analysis and visualization of functional magnetic resonance neuroimages. Comput Biomed Res. 1996;29:162-73.

28. Jenkinson M, Beckmann CF, Behrens TE, Woolrich MW, Smith SM. Fsl. Neuroimage. 2012;62:782-90.

29. Stern ER, Welsh RC, Fitzgerald KD, Gehring WJ, Lister JJ, Himle JA, et al. Hyperactive error responses and altered connectivity in ventromedial and frontoinsular cortices in obsessive-compulsive disorder. Biol Psychiatry. 2011;69:583-91.

30. Taylor SF, Martis B, Fitzgerald KD, Welsh RC, Abelson JL, Liberzon I, et al. Medial frontal cortex activity and loss-related responses to errors. J Neurosci. 2006; 26:4063-70.

31. Ongur D, Ferry AT, Price JL. Architectonic subdivision of the human orbital and medial prefrontal cortex. J Comp Neurol. 2003;460:425-49.

32. Monosov IE, Hikosaka O. Regionally distinct processing of rewards and punishments by the primate ventromedial prefrontal cortex. J Neurosci. 2012;32:10318-30.

33. Mackey S, Petrides M. Quantitative demonstration of comparable architectonic areas within the ventromedial and lateral orbital frontal cortex in the human and the macaque monkey brains. European Journal of Neuroscience. 2010;32:1940-50.

34. Wallis CU, Cockcroft GJ, Cardinal RN, Roberts AC, Clarke HF Hippocampal interaction with area 25 , but not area 32 , regulates marmoset approach-avoidance behavior. Cereb Cortex. 2019.

35. Hofmann SG, Bitran S. Sensory-processing sensitivity in social anxiety disorder: relationship to harm avoidance and diagnostic subtypes. J Anxiety Disord. 2007; 21:944-54.

36. Abrams KY, Yune SK, Kim SJ, Jeon HJ, Han SJ, Hwang J, et al. Trait and state aspects of harm avoidance and its implication for treatment in major depressive disorder, dysthymic disorder, and depressive personality disorder. Psychiatry Clin Neurosci. 2004;58:240-8.

37. Wong JJ, O'Daly O, Mehta MA, Young AH, Stone JM. Ketamine modulates subgenual cingulate connectivity with the memory-related neural circuit-a mechanism of relevance to resistant depression? PeerJ. 2016;4:e1710.

38. Carlson PJ, Diazgranados N, Nugent AC, Ibrahim L, Luckenbaugh DA, Brutsche N, et al. Neural correlates of rapid antidepressant response to ketamine in treatment-resistant unipolar depression: a preliminary positron emission tomography study. Biol Psychiatry. 2013;73:1213-21.

39. Deakin JF, Lees J, McKie S, Hallak JE, Williams SR, Dursun SM. Glutamate and the neural basis of the subjective effects of ketamine: a pharmaco-magnetic resonance imaging study. Arch Gen Psychiatry. 2008;65:154-64.

40. Downey D, Dutta A, McKie S, Dawson GR, Dourish CT, Craig K, et al. Comparing the actions of lanicemine and ketamine in depression: key role of the anterior cingulate. Eur Neuropsychopharmacol. 2016;26:994-1003.

41. Skinner B. The Behavior of Organisms: an experimental analysi. New York, London Appleton-Century Company. 1938.

42. Hull C. Principles of Behavior, an Introduction to Behavior Theory. New YorkD Appleton-Century Company. 1943.

43. Murrough JW, Perez AM, Pillemer S, Stern J, Parides MK, aan het Rot M, et al. Rapid and longer-term antidepressant effects of repeated ketamine infusions in treatment-resistant major depression. Biol Psychiatry. 2013;74:250-6. 